\title{
DIFERENÇAS SEXUAIS DA ATENÇÃO VISUAL NA EPILEPSIA PARCIAL CRIPTOGÊNICA
}

\author{
PAULO AFONSO MEDEIROS KANDA*, CARLOS JOSÉ REIS DE CAMPOS**
}

\begin{abstract}
RESUMO - Estudamos o rendimento em testes de atenção visual verbal e não-verbal de 14 homens e 18 mulheres controles normais e 33 pacientes com epilepsia parcial criptogênica. O grupo epiléptico foi constituído de 17 homens e 16 mulheres sem evidência de lesões à tomografia computadorizada de crânio. As descargas epilépticas interictais localizavam-se unilateralmente, no lobo temporal direito ( 8 homens, 8 mulheres) ou no esquerdo ( 9 homens, 8 mulheres). Odesempenho dos homens epilépticos foi semelhante ao dos homens controles. Por outro lado, o rendimento das mulheres epilépticas foi pior que o das mulheres controles. Estes resultados parecem de acordo com a literatura que sugere que a atenção visual nas mulheres dependa da integridade funcional dos dois hemisférios cerebrais. Os homens processariam basicamente os estímulos visuais verbais no hemisfério cerebral esquerdo e os não-verbais no hemisfério cerebral direito. São necessários estudos posteriores para o melhor entendimento dessas diferenças sexuais da assimetria funcional hemisférica.
\end{abstract}

PALAVRAS CHAVE: diferenças sexuais, atenção visual, epilepsia parcial.

\section{Sexual differences of visual attention in cryptogenic partial epilepsy}

SUMMARY - The performance in visual verbal and non-verbal attentional tests was studied in 14 male and 18 female control subjects and 33 patients with cryptogenic partial epilepsy. The epileptic group was formed of 17 men and 16 women with no evidence of brain damage in CT scan examinations. Interictal epileptiform activity was observed only unilaterally, either in the right ( $8 \mathrm{male}, 8 \mathrm{female}$ ) or left ( 9 male, $8 \mathrm{female}$ ) temporal lobes. The performance of epileptic men in visual tests was similar to that of normal men. On the other hand, the performance of epileptic women was worse than that of normal women. These results seem in accordance with the literature that suggests that visual attention in women depends on the functional integrity of both cerebral hemispheres. In men, verbal visual attention is suggested to occur in the left cerebral hemisphere while non-verbal processes, predominantly in the right cerebral hemisphere. Further studies are necessary for the understanding of these sexual differences in cerebral hemispheric asymmetry.

KEY WORDS: sexual differences, visual attention, partial epilepsy.

Luria denomina atenção à função resultante dos processos mentais que permitem ao indivíduo selecionar e direcionar-se para determinados estímulos sensoriais, sendo o canal visual classicamente usado nos testes de avaliação cognitiva ${ }^{10,11}$. Pelo estudo da atenção podemos conhecer melhor os mecanismos funcionais que modulam o desempenho do sistema nervoso central (SNC) e entender processos relacionados aos mais diversos distúrbios funcionais, desde estados confusionais até a dificuldade de aprendizagem escolar ${ }^{6,23}$. Alterações cognitivas transitórias são bem descritas nas epilepsias generalizadas como, por exemplo, na epilepsia generalizada ausência ${ }^{21}$. Poucos estudos têm analisado especificamente a atenção em pacientes com epilepsia parcial ${ }^{19,13}$. Os pacientes com epilepsia parcial possibilitam a análise de funções relacionadas às epilepsias e à atenção, especialmente no que tange à assimetria funcional hemisférica ${ }^{13,14,17}$.

Setor de Investigação e Tratamento das Epilepsias (SITE), Disciplina de Neurologia, Escola Paulista de Medicina: *Mestrando em Neurologia; **Professor Adjunto Doutor, Chefe do SITE. Aceite: 28-julho-1993.

Dr. Carlos José Reis de Campos - Disciplina de Neurologia (SITE), Escola Paulista de Medicina - Rua Botucatu 740 - 04023-900 São Paulo SP - Brasil. 
Como vem sendo descrita na literatura a existência de diferenças sexuais na assimetria funcional hemisférica ${ }^{19}$, o propósito deste estudo foi analisar a influência sexual e da lateralização do foco epiléptico ao EEG no desempenho de provas para atenção visual em pacientes com epilepsia parcial criptogênica.

\section{CASUÍSTICA E METODOLOGIA}

O Grupo Epiléptico foi constituído de 17 homens e 16 mulheres destros, com mediana de idade de 28 anos, sendo $52 \%$ ( $n=17)$ do sexo masculino e $48 \%(n=16)$ do sexo feminino. Cada paciente foi submetido a um mínimo de dois eletrencefalogramas intercríticos mostrando descargas epilépticas focais, sempre unilaterais, evidentes e de projeção sempre no mesmo lobo temporal para cada paciente. Os casos com lesão à tomografia computadorizada de crânio (TCC) foram excluídos. Os pacientes do Grupo Epiléptico apresentaram 15 anos como média de idade do início da epilepsia ( $54 \%$ dos casos) e duração da epilepsia superior a 5 anos em $66 \%$ dos casos. Destes pacientes, 16 (48\%) apresentavam crises pouco frequentes (menos de 1 crise por mês), 17 (52\%) crises frequentes ( 1 crise por mês) e nenhum apresentou crises muito frequentes (mais de 1 crise por mês).

O Grupo Controle constituiu-se de 14 homens e 18 mulheres com mediana de idade de 32 anos. Este grupo, de mesmo nível sócio-econômico, foi selecionado dentre acompanhantes e familiares dos pacientes. Não apresentavam história de doenças neurologicas ou uso de drogas estimulantes ou depressoras do SNC.

Foram estudados apenas indivíduos com exame neurológico normal, escolaridade mínima de quarto ano primário e avaliação de função visual (acuidade e campimetria) normal. Os pacientes apresentavam história de mais de dois anos de crises epilépticas parciais com generalização subsequente e faziam uso das drogas antiepilépticas (DAE) nas seguintes doses diárias:17 pacientes, carbamazepina (CBZ) 600mg/dia; 10 pacientes, fenobarbital (FB) $100 \mathrm{mg} / \mathrm{dia} ; 4$ pacientes, CBZ 500mg/dia + FB $100 \mathrm{mg} / \mathrm{dia} ; 2$ pacientes, FB $100 \mathrm{mg} /$ dia + hidantoina $200 \mathrm{mg} / \mathrm{dia}$.

Tanto as mulheres controles quanto as pacientes epilépticas apresentavam escolaridade de 6 anos em média. Apenas uma paciente chegou a completar o primeiro ano do segundo grau. Apenas três mulheres dentre 18 do grupo controle concluiram a terceira série do segundo grau.

Aplicamos 4 testes visuais de cancelamento. As folhas de teste mediam $29 \times 36 \mathrm{~cm}$ apresentando 374 sígnos dos quais 60 deveriam ser circulados a caneta pelo paciente. Dois tipos de erros eram possíveis: por omissão $(O)$ do sígno a ser cancelado e por troca $(T)$ quando da escolha de signo errado. Os testes foram divididos em dois grupos de

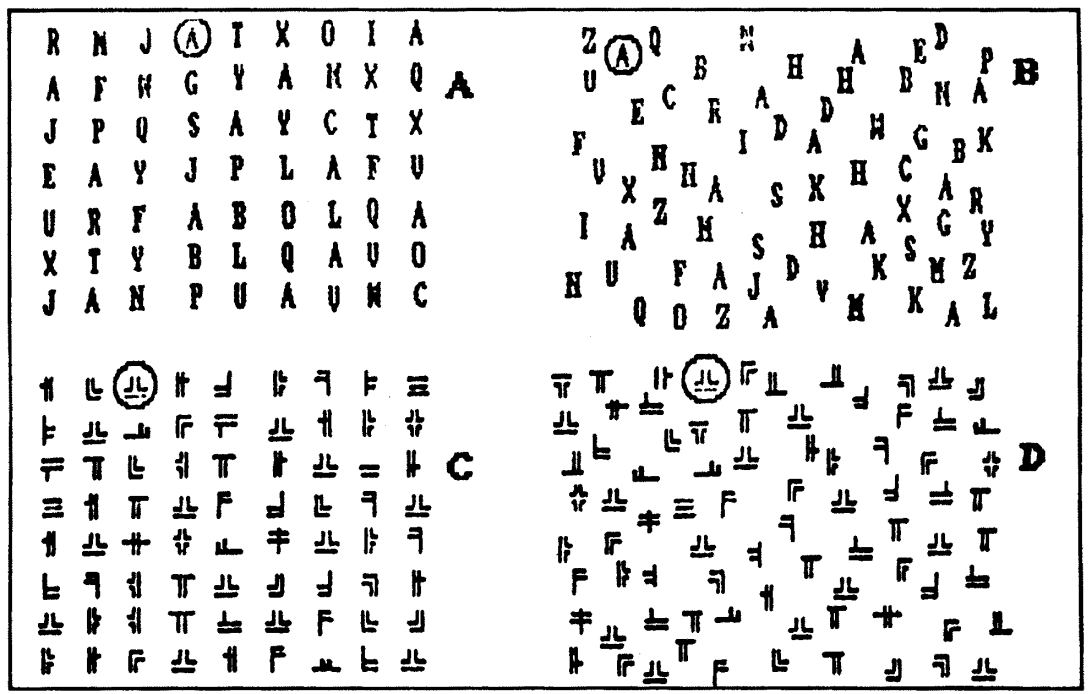

Fig 1. Fragmentos dos testes de atenção visual. A, teste verbal ordenado (linhas e colunas); $B$, teste verbal aleatório; $C$, teste não-verbal ordenado; $D$, teste não-verbal aleatório. $O$ paciente é orientado a marcar com a caneta toda vez que encontrar o signo exemplificado, terminando $o$ teste o mais rápido possível. 
Tabela 1. Erros por omissão (0) versus troca $(T)$ para os testes visuais.

\begin{tabular}{|c|c|c|c|c|c|c|c|c|c|c|c|c|}
\hline \multirow{5}{*}{ Testes } & \multicolumn{4}{|c|}{ Controles } & \multicolumn{8}{|c|}{ Foco Temporal } \\
\hline & \multirow{2}{*}{\multicolumn{2}{|c|}{$\mathbf{M}$}} & \multirow{2}{*}{\multicolumn{2}{|c|}{$\mathrm{F}$}} & \multicolumn{4}{|c|}{ Direito } & \multicolumn{4}{|c|}{ Esquerdo } \\
\hline & & & & & \multicolumn{2}{|c|}{$\mathbf{M}$} & \multicolumn{2}{|c|}{$\mathbf{F}$} & \multicolumn{2}{|c|}{$\mathbf{M}$} & \multicolumn{2}{|c|}{$\mathbf{F}$} \\
\hline & \multicolumn{2}{|c|}{$(\mathrm{N}=16)$} & \multicolumn{2}{|c|}{$(\mathrm{N}=16)$} & \multicolumn{2}{|c|}{$(\mathrm{N}=8)$} & \multicolumn{2}{|c|}{$(\mathrm{N}=8)$} & \multicolumn{2}{|c|}{$(\mathrm{N}=9)$} & \multicolumn{2}{|c|}{$(\mathrm{N}=8)$} \\
\hline & 0 & $\mathbf{T}$ & 0 & $T$ & $\mathrm{O}$ & $\mathrm{T}$ & 0 & $\mathrm{~T}$ & $\mathrm{O}$ & $\mathbf{T}$ & $\mathrm{O}$ & $\mathrm{T}$ \\
\hline $\begin{array}{l}\text { Verbal } \\
\text { ordenado }\end{array}$ & \multicolumn{2}{|c|}{ N.S. } & 1,4 & $0,3^{*}$ & 1 & 0* & 1 & $0^{*}$ & \multicolumn{2}{|c|}{ N.S. } & \multicolumn{2}{|c|}{ N.S. } \\
\hline $\begin{array}{l}\text { Verbal } \\
\text { aleatório }\end{array}$ & 3 & $0^{*}$ & 2 & $0^{*}$ & 1 & $0^{*}$ & 3 & $0^{*}$ & 3 & $0^{*}$ & 4 & $0^{*}$ \\
\hline $\begin{array}{l}\text { Não-verbal } \\
\text { ordenado }\end{array}$ & 6 & $2^{*}$ & 6 & $1^{*}$ & \multicolumn{2}{|c|}{ N.S. } & \multicolumn{2}{|c|}{ N.S. } & \multicolumn{2}{|c|}{ N.S. } & \multicolumn{2}{|c|}{ N.S. } \\
\hline $\begin{array}{l}\text { Não-verbal } \\
\text { aleatório }\end{array}$ & \multicolumn{2}{|c|}{ N.S. } & 7 & $3 *$ & 5 & $3 *$ & 12,6 & $1^{*}$ & 10 & $2,4^{*}$ & 12 & $2^{*}$ \\
\hline
\end{tabular}

Os dados da tabela mostram o número de erros.

Teste de Wilcoxon. M, masculino; F, feminino; * $\mathrm{p}<0,05$; N.S., não significativo.

testes visuais, verbais e não-verbais. Os estímulos verbais estão relacionados à linguagem escrita e oral, enquanto os estímulos não-verbais relacionam-se à orientação vísuo-espacial, à interpretação de sinais, desenhos e gráficos sem caráter linguístico. Estes testes foram apresentados de dois modos quanto à distribuição dos caracteres: ordenado em linhas e colunas e de maneira aleatória (Fig 1, Mesulam, 1988, modificada).

\section{RESULTADOS}

Os homens com epilepsia parcial com foco de projeção temporal, tanto à direita quanto à esquerda, não apresentaram diferenças estatisticas do desempenho nos testes visuais verbais e não-verbais em relação aos seus controles. Além disso, os pacientes do sexo masculino usando DAE não apresentaram diferenças de rendimento em relação aos seus controles não medicados. Pacientes de ambos sexos em uso de carbamazepina não apresentaram diferenças no rendimento em relação aos que usavam fenobarbital.

Tanto homens como mulheres, pacientes epilépticos e controles, apresentaram predomínio significativo de erros por omissão em relação aos erros por troca, nos testes visuais verbais e não-verbais de cancelamento. Além disso, nos pacientes epilépticos e controles, de ambos os sexos, os testes aleatórios foram mais sensíveis, evidenciando melhor as diferenças entre omissões e trocas (Tabela 1).

Tabela 2. Tempo de duração dos testes visuais.

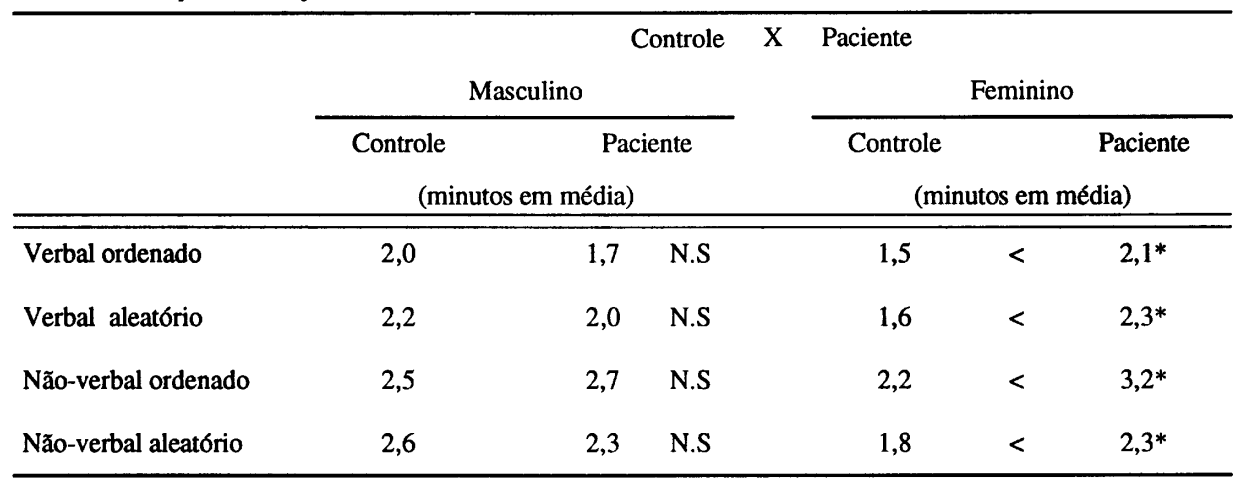

Teste de Mann-Whitney. ${ }^{*} \mathrm{p}<0,05 ;$ N.S., não significativo. 
As mulheres do grupo epiléptico (com descargas tanto no lobo temporal direito quanto no esquerdo) apresentaram mais erros (teste de Mann-Whitney, alfa $=5 \%$ ) por omissão (média de erros $=12$ ) no teste aleatório não-verbal que seus controles (média de erros $=7$ ) (Tabela 1) e demoraram mais que seus controles para realizar todos os testes (Tabela 2).

\section{COMENTÁRIOS}

Vários fatores podem influir no desempenho dos pacientes com epilepsia nos testes de atenção. Tem sido sugerido na literatura que pacientes com crises generalizadas ou com generalização subseqüente, quando submetidos a testes de atenção, têm desempenho aquém do esperado ${ }^{7}$. Em nosso estudo observamos que apenas as mulheres com epilepsia parcial criptogênica tiveram rendimento inferior aos seus controles, fato não observado entre os homens.

Nossos achados provavelmente divergem de outros da literatura por diferenças de casuística. As epilepsias associadas a lesões cerebrais costumam apresentar formas mais graves que as epilepsias criptogênicas. Assim, quando grupos heterogêneos de pacientes (com e sem lesão cerebral) são analisados em conjunto, a interpretação dos resultados pode ser variável ${ }^{5,17}$. Nós estudamos exclusivamente um grupo de pacientes com formas mais leves de epilepsia parcial criptogênica.

O uso de DAE é outro fator amplamente discutido na literatura como causa de déficit cognitivo ${ }^{4}$. Entretanto, mesmo em uso de DAE, nossos pacientes masculinos não tiveram rendimento pior que seus controles. Acreditamos que isto ocorreu porque nossos pacientes apresentavam crises pouco frequentes, de fácil controle, em uso de doses terapêuticas baixas de DAE. Possivelmente outros fatores como politerapia, gravidade da síndrome epiléptica, frequência das crises e doenças associadas sejam mais importantes para produzir distúrbio de "atenção" que o uso crônico de DAE em doses terapêuticas baixas.

O fato de nossos pacientes e controles, masculinos e femininos, errarem mais por omissão que por troca está de acordo com outros relatos na literatura ${ }^{2,3,12}$. Além disso, os testes aleatórios, por serem mais difíceis, tornaram ainda mais evidente essa diferença entre erros por omissão versus erros por troca.

As mulheres epilépticas tiveram desempenho pior que seus controles em todos os testes verbais e não-verbais. Os pacientes masculinos tiveram rendimento semelhante aos controles. Isto nos leva a considerar para cada sexo a existência de uma forma peculiar de processamento da atenção visual. É referido na literatura que indivíduos destros processam estímulos verbais primordialmente por meio do hemisfério cerebral esquerdo e os não-verbais por meio do hemisfério cerebral direito ${ }^{8,24} \mathrm{e}$ que as mulheres processam estímulos verbais mais difusamente que os homens, utilizando os dois hemisférios cerebrais ${ }^{15,20}$.

Nossos achados podem apoiar a teoria de que as mulheres parecem processar também a informação visual não-verbal usando simultaneamente os dois hemisférios cerebrais ${ }^{19,22}$. $\mathrm{O}$ desempenho pior das mulheres epilépticas em relação aos demais grupos (inclusive ao grupo dos homens epilépticos) pode sugerir que o foco epiléptico à direita ou à esquerda tenha interferido neste possível modo peculiar de processamento cognitivo no sexo feminino. Assim sendo, podemos considerar que o foco epiléptico foi apenas mais um elemento de interferência, acentuando o padrão de resposta esperado para sexo feminino. Ressaltamos que são necessários estudos posteriores para o melhor entendimento dessas diferenças sexuais da assimetria funcional hemisférica.

\section{REFERÊNCIAS}

1. Aarts HP, Binnie CD, Smith AM, Wilkings AJ. Selective cognitive impairment during focal and generalized epileptiform EEG activity. Brain 1984, 107: 293-308.

2. Bowers D, Heilman KM. Pseudoneglect: effects of hemispace on a tactile line bisection task. Neuropsychol 1980, 18:491-498.

3. Bradshaw JL, Nettleton NC, Nathan G, Wilson LE. Bisecting rods and lines: effects of horizontal and vertical posture on left-side underestimation by normal subjects. Neuropsychol 1985, 23:421-425. 
4. Browne TR, Feldman RG: Epilepsy: diagnosis and management. Boston: Little Brown, 1983.

5. Camfield PR, Gates R, Ronen G, Camfield C, Fergunson A, MacDonald GW. Comparison of cognitive ability, personality, profile, and school success in epileptic children with pure rigth versus left temporal lobe EEG. Ann Neurol 1984, 15:122-126.

6. Chedru F, Geschwind N. Disorders of higher cortical functions in acute confusional states. Cortex 1972, 8:395-411. 7. Giordani B, Berent S, Sackellares JC, Rourke D, Seidenberg S, O'Leary DS, Dreifuss FE, Boll TJ. Intelligence test performance of patients with partial and generalized seizures. Epilepsia 1985, 26: 37-42.

8. Kimura D. Funcional asymmetry of the brain in dichotic listening. Cortex 1967, 3:395-396.

9. Ladavas E, Umilta C, Provinciali L. Hemisphere-dependent cognitive performances in epileptic patients. Epilepsia 1979, 20: 493-502.

10. Luria AR. Higher cortical function in man. New York: Basic Books, 1966.

11. Luria, AR. El cerebro en accion. Barcelona Fontanella, 1979.

12. Mark VW, Heilman KM. Bodily neglect and orientational biases in unilateral neglect syndrome and normal subjects. Neurology 1990, 40: 640-643.

13. Mazzucchi A, Parma M. Responses to dichotic listening in temporal epileptic with and without lesions. Cortex 1978, 14: 381-390.

14. Mazzucchi A, Visintini D, Magnani G, Cattelani R, Parma M. Hemispheric prevalence changes in partial epileptic patients on perceptual and attentional tasks. Epilepsia 1985, 26:379-390.

15. McGee MG. Human spatial, psychometric studies and envirommental, genetic, hormonal and neurological influences. PSYCHOL BULL 1979, 86: 889-918.

16. McGlone J. Sex differences in human brain asymmetry. Behav Brain Sci 1980 3: 215-263.

17. McIntyre M, Pritchard PB, Lombroso CT. Left and right temporal lobe epileptic: a controlled investigation of some psychological differences. Epilepsia 1976, 17: 377-386.

18. Mesulam MM. Principles of behavioral neurology. Philadelphia: Davis, 1988.

19. Milstein V, Small IF, Malloy FW, Small JG. Influence of sex and handedness of hemispheric funcioning. Cortex 1979, 15:439-449.

20. Muszkat M, Frayman L, Vincenzo NS, Campos CJR. Diferenças Sexuais na assimetria funcional hemisférica. Arq Neuropsiquiatr 1991, 49: 142-146.

21. Niedermeyer E. The epilepsies: diagnosis and management. Baltimore: Urban \& Schwarzenberg, 1990.

22. Segalowitz SJ. Two sides of the brain: brain lateralization explored. New Jersey: Spectrum, 1983.

23. Silverman J. Problems of attention in research and theory in schizophrenia. Psychol Rev 1964, 71:352-379.

24. Springer SP, Deutsch G. Left brain-right brain. New York: Freeman, 1984. 